\title{
The research on rainwater power generation system
}

\author{
Zhicheng Huang \\ School of North China Electric Power University, Baoding 071000, China; \\ 648277489@qq.com
}

\begin{abstract}
Keywords: Fluent simulation, Motor distribution model, Fluid mechanics, Power electronic technology.
\end{abstract}

\begin{abstract}
The potential energy of rain has certain potential in power generation. This article used the technology of cross flow rainwater power generation. In order to achieve the highest efficiency of rainwater power generation, this paper established a reasonable distribution model of generator in pipeline by the theory of fluid mechanics and the simulation of the generation process of AC generator to obtain the height difference of the optimal generator distribution and the optimal height of water storage. Rational distribution of electric energy can be achieved by electric power storage and power grid connected through power electronic technology. In the end, this paper not only estimated the economic performance of rainwater power generation, but also proposed the development trend of the future rainwater power generation technology.
\end{abstract}

\section{Introduction}

At present, the effective utilization of clean energy has become a hot research topic. Both at home and abroad, researches on the use of rainwater have a long history, but most rainwater resources are used for collection and reuse to save water resources, and reduce the role of municipal pressure. Rainwater power generation can not only save part of energy, but also effectively solve ground collapse problem caused by the impact of rainstorm. At present some proposals about rainwater power generation technology were put forward in China, and some scholars have carried out part of research on the design of rainwater generator. However, there is no in-depth study about the integrated rainwater power generating system and its optimization design. This paper used the technology of cross flow rainwater power generation that comes from literature [1].For realizing the best use of potential rainwater energy, a reasonable distribution model of generator in pipeline was established based on the research of the micro turbine fan blade in the literature [2].Energy storage and power grid connected was achieved by using the technology of Rectifier voltage regulator and the phase-locked loop that comes from literature [3].

\section{Design scheme of rainwater power generation system}

Considering the rich rainfall and some very tall buildings in Southern China south, the potential energy of rain has certain potential in power generation. If we supply power for small power appliances with the electricity that generated by the potential energy of rain, we can not only conserve energy and reduce emissions, but also effectively solve ground collapse problem caused by the impact of rainstorm. At the same time, the reuse of rainwater is not affected. This paper describes the scheme from four aspects: the rainwater collection device, the generator distribution model, the electric energy storage, and the feasibility and the development direction.

\subsection{Rainwater collecting device}

In order to make the flow of rainwater in pipeline is relatively constant, generator speed fluctuation is small, so at the top of the floor floater type rainwater collection system is set.The rain goes through a strainer before it flows into the reservoir to prevent large particulate matter from entering the pipe damaged motor fan blade. In order to collect all the rain on the roof, the rainwater collecting device was set in the bottom of the roof slope, which above the drainage pipe was embedded in the ground. 
When the rain reaches a certain height, the pipe pens because of the buoyancy effect. Then the rainwater pours down and its potential energy turns into the electric energy.

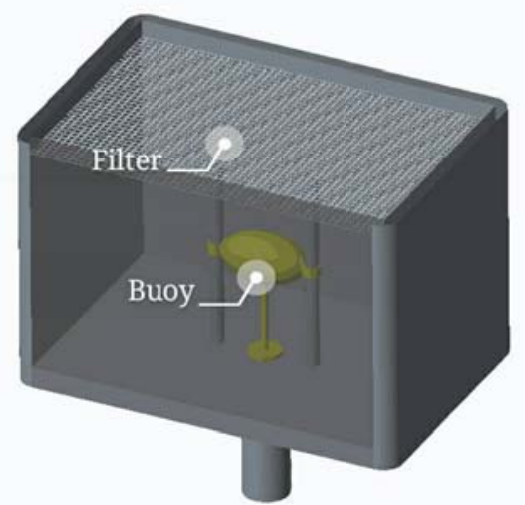

\subsection{Generator distribution model}

Fig1:Rainwater collecting device

In this paper, fluent simulation is used to test the rationality of the model. Using fluent6.3.2 as a numerical simulation tool, two dimensional unsteady solver, pressure based model, the standard $k-\varepsilon$ turbulence mode are selected. Gravity is considered and the settings for the remaining parameters are the default values.

Table 1 Model related variables

\begin{tabular}{cc}
\hline Symbol & Definition \\
\hline$\omega$ & The height between the first motor and the bottom of the buoy \\
$H_{n}$ & Optimum generator speed under $H_{n}$ condition \\
$w_{n}$ & The size of pressure that the first generator's fan blade bear \\
$F_{n}$ & The amount of energy the first generator can emit by one time \\
$W_{n}$ & displacement of water \\
$v_{n}$ & Energy utilization efficiency of the first generator \\
$t_{n}$ & The time of once amount of water pass by the first generator \\
$\mathrm{R}$ & Fan blade radius of micro generator \\
$S_{m}$ & Minimum water flow cross sectional area between motor and pipe wall \\
$v$ & Flow velocity \\
$\mathrm{h}$ & Height of water storage \\
$H_{Z}$ & Height difference between generators \\
\hline
\end{tabular}

The pressure of the water flow on the generator fan blade is different because of different speeds of the generator. And the pressure of the water flow on the fan blade of the generator is proportional to $H_{n}$. So, taking $H_{1} H_{2} \ldots H_{10}=36 \ldots 30$ meters as the vertical height of the first generator. Then, the relationship between $\omega$ and $F_{n}$ under different height conditions is simulated. The results show that the relationship between the two is as follows:

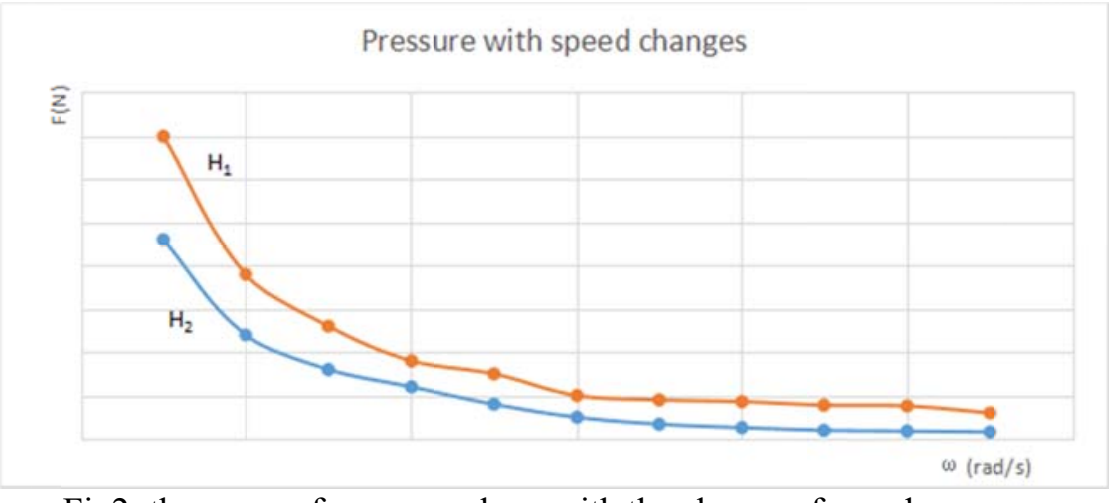

Fig2: the curve of pressure along with the change of speed 


$$
W_{n}=\int_{0}^{R} F_{n} * \mathrm{w} d r * t_{n}
$$

The amount of work done by one time displacement on the motor can be calculated by using formula (1).

Assume that the generator is accelerated quickly, and the final speed of generator is $\omega$. Since the linear velocity of generator fan blade is not greater than the velocity of the water flow, this paper assumes the velocity of the water flow in the position of $S_{m}=v_{1} \approx 1.1 \omega \mathrm{R}$. Then the initial velocity of water flow at the top of pipe can be obtained by using the continuities theorem. In the simulation of this paper, assuming that the quantity of one time displacement is $1 \mathrm{~m}^{3}$,pipe diameter is $0.5 \mathrm{~m}$, generator fan blade radius is $0.4 \mathrm{~m}, \mathrm{~A} t_{n}$ can be obtained by means of a $\omega$, the $W_{n}$ corresponding to the $\omega$ and the $t_{n}$ can be obtained.

The relationship between $\omega$ and $W_{n}$ under different heights was further obtained, and their relationship image is as follows:

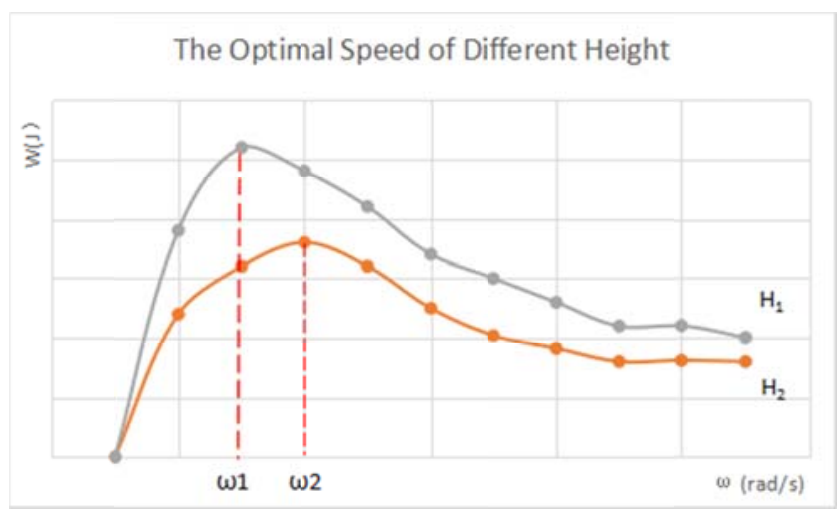

Fig3:Optimum speed at different heights

From the picture 3, it can be seen that one $w_{n}$ corresponds to one height, and at that point, power generation efficiency reaches the highest.

$$
v_{n}=\frac{W_{n}}{\operatorname{mg} H_{n}}
$$

The relationship between $v_{n}$ and $\mathrm{H}$ was further obtained by using the equation (2), and their relationship image is as follows:

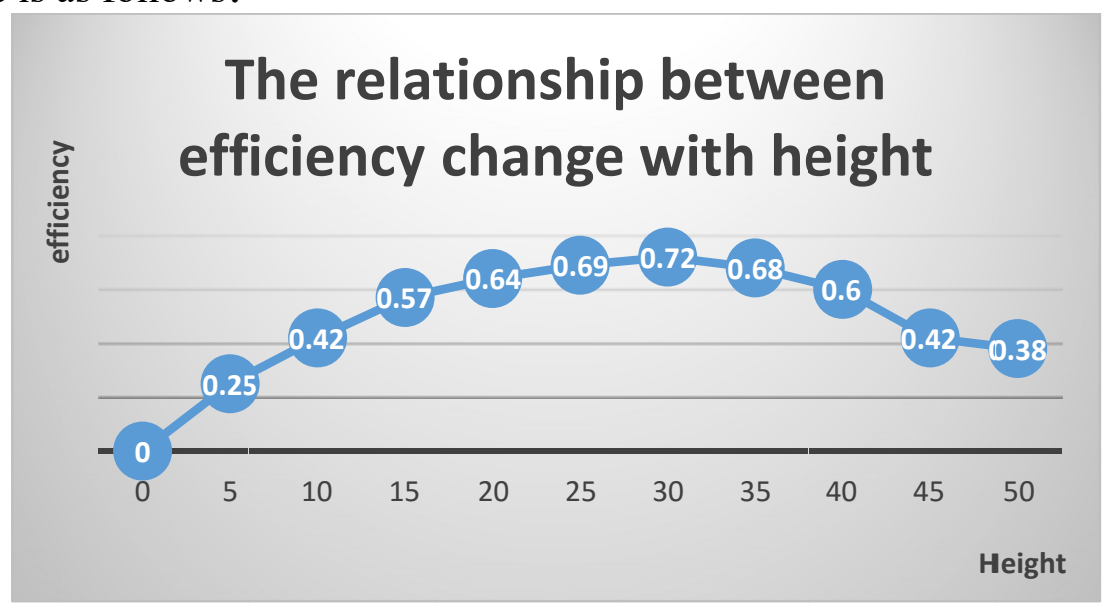

Fig4: The efficiency curve with the change of height

From the picture above, the optimum efficiency can be easily obtained and the corresponding $w_{n}$ and $W_{n \max }$ can be further got. Under this condition, it is known that the utilization rate of rainwater potential energy is the maximum. From the above, the initial velocity of the full pipe flow can be obtained by using $w_{n}$. According to the energy equation: $z_{1}+\frac{p_{1}}{\rho g}+\frac{u_{1}^{2}}{2 g}=z_{2}+\frac{p_{2}}{\rho g}+\frac{u_{2}^{2}}{2 g}+$ $h_{w}$.Among them, $z_{1}, z_{2}$ were water source location. $p_{1}, p_{2}$ were pressure head, $\frac{u_{1}^{2}}{2 g} 、 \frac{u_{2}^{2}}{2 g}$ wereVelocity head, and $h_{w}$ was head loss. Obviously, this problem can be described as the position of head and $\mathrm{H}$ 
can be converted to how much the flow velocity head. Assume that Velocity coefficient $=1.0, h_{w}=0$, then the initial velocity of the full pipe flow $=\sqrt{2 g h}$.

Then, further to obtain the best water storage height $\mathrm{H}$.

By the previous work, the optimal vertical height between the first motor and the bottom of the float can be obtained. The distribution of the following motors is based on the principle of equivalence. According to the continuity theorem, the flow velocity is approximately constant under the condition of the full pipe flow. So we only need to compensate for the loss of mechanical energy after the water flow through the first motor.

$$
W_{\text {nmax }}=\operatorname{mgH}_{z}^{0}
$$

Since other losses are unknown, total loss of flow mechanical energy $\mathrm{W}$ can be obtained by using the generator power $W_{n \max }$ multiplied by a coefficient a.The $\mathrm{W}=\mathrm{mg} H_{Z}$ can realize the compensation for the loss of the mechanical energy of the water flow.

Once again use the simulation to determine the coefficient $a$. and the distance between the second motor and the first motor $=\mathrm{H}_{z}^{0 *} \mathrm{a}$ is only needed to be made. In the simulations, try different a to make the pressure of the second motor fan blades can be roughly the same as the first one.

The simulation can determine the $\mathrm{a} \approx 1.37$

\subsection{Electric energy storage}

This paper describes utilizing the electricity generated from rainwater, based on the related theory of power and electron technology, to store electric power and complete the grid connection. 24V, D.C. can be obtained to store electric power for the secondary cell through the single-phase uncontrollable-bridge commutated circuit of capacitor filtering as well as the serial feedback regulating circuit. As for the electricity which is surplus to the storage battery, by means of voltage booster circuit, full-bridge circuit of single-phase voltage type, SPWM technology and phase-locked loops technology, the results that alternating current in basic accord with the voltage-frequency and phase position of electric network will be realized, then, so is it with stable interconnection. But taking the cost factor into consideration, it is able to charge the solar battery with the electricity as the supplementary to photovoltaic power generation. Then, the part that's combined to the grid will be moved onto the electrical power grid together with the photovoltaic power generation.

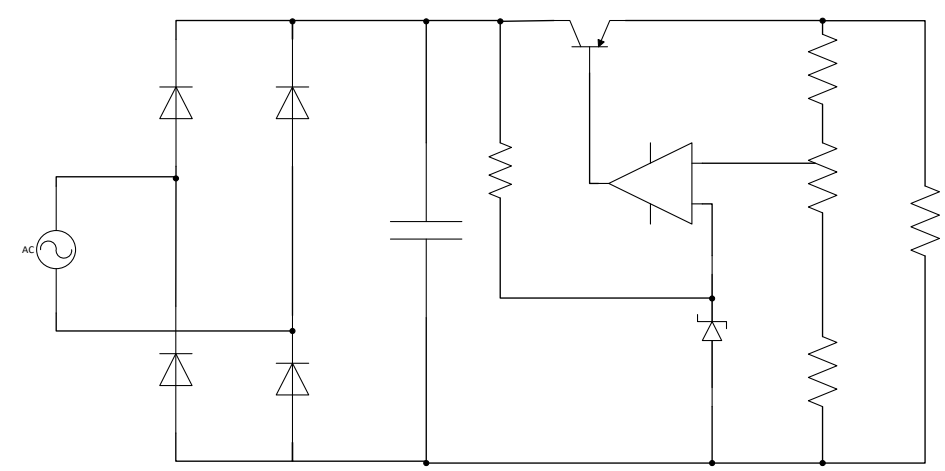

Fig 5: Rectifying and voltage-stabilizing battery circuit

\subsection{The analysis of feasibility and perspective from rainwater-generated electricity}

From all models above, we know, under the assumed conditions mentioned above, the optimum one is near the place that $\mathrm{H}=30 \mathrm{~m}, \mathrm{~Hz}=29.6$ and the water-storage height is equal to $1.8 \mathrm{~m}$, where the transformation efficiency of rainwater's gravitational potential energy can reach $72 \%$. In the light of actual conditions here, taking Guangxi province as an example, whose yearly precipitation can reach nearly $1800 \mathrm{~mm}$, suppose that one domestic story with an area of $500 \mathrm{~m}^{2}$ and a height of $120 \mathrm{~m}$ will be equipped with four electric generators. What's more, per micro hydroelectric generator costs 500yuan, the whole commutating and voltage-stabilizing circuit and the wire splices costs $400 y u a n$, and the cost of a storage battery is 2000 and building one is 1000yuan. And per kilowatt hour needs 55 fen. So the time to recover the costs is at least 33 years under those conditions. Thereupon, it is unpractical and inapplicable in general. However, in consideration of using a solar cell directly, it will save costs. Thus it will take only 20 years if using rainwater to supplement the solar electrical energy generation. 
For as much as that stored water can flow down and be spread to irrigate the community garden, it is not only beneficial to economizing water resource, but also to lightening the possibility effectively that the ground may to collapse due to the shorts to the ground caused by drainage from high level, and therefore, rainwater generation, to a certain extent, has a great foreground.

\section{Summary}

This paper studies the relevant issues of rainwater generation in terms of rainwater generation system.

The optimal revenue model structured by modeling and simulation techniques has a strong versatility. That means, it's prone to get the optimal altitude differences of the electric motor's distribution and the optimum height of water-storage that matched those being up to the practical diameter of the pipe and semidiameter of the electric engine fan. It is an effective way to store and parallel in electric energy by using rectifying and voltage-stabilizing technology and inverter power source circuit. From the perspective of the benefits of rainwater generation, the economical benefit that simply comes from the rainwater generator system is greatly limited. But if treated as the supplementary to solar energy to further realize hybrid power generation, it will achieve the reduction of costs and a certain economic benefit. The rainwater generator system can lighten the shock, caused by the torrential rainfall from high level, to the ground, and can be spread to irritate green land. Generally speaking, as a supportive equipment, this system is fairly promising.

\section{Reference}

[1] Xu Dongwei, Ning houfei, Zhang yongbin,et al. A new technology of high rise rainwater power generation based on the concept of cross flow generation. SHANXI ARCHITECTURE.Vol.40 No.31 Nov. 2014.

[2] GaoZhan, Chen,Meijie, AnCheng,et al. Flow field of Numerical simulation research on Composite water power generating device for micro hydro turbine. ENE R GY CONSE R VATION TECHNOLOGY. Vol. 31, Sum. No. 182 November. 2013, No. 6.

[3] ZhaoWei: Research on Grid-Connection Photoboltaic System(Doctor of Engineering, Hefei University of Technology,China,2003).p.53.

[4] Shi Xinchun, Wang Yi,Sun Liling,et al. Power electronic technology. Higher Education Press,2013,p47-50. 